\title{
Assessment of non-invasive ICP during CSF infusion test: an approach with transcranial Doppler
}

\author{
D. Cardim ${ }^{1} \cdot$ M. Czosnyka ${ }^{1,5} \cdot$ J. Donnelly $^{1} \cdot$ C. Robba $^{2}$ - B. C. T. Cabella ${ }^{1} \cdot$ X. Liu ${ }^{1}$. \\ M. T. Cabeleira ${ }^{1}$ - P. Smielewsky ${ }^{1}$ - C. Haubrich ${ }^{3}$ - M. R. Garnett ${ }^{4}$ - J. D. Pickard ${ }^{4}$. \\ Z. Czosnyka ${ }^{1}$
}

Received: 6 October 2015 / Accepted: 2 December 2015 / Published online: 23 December 2015

(C) The Author(s) 2015. This article is published with open access at Springerlink.com

\begin{abstract}
Background This study aimed to compare four non-invasive intracranial pressure (nICP) methods in a prospective cohort of hydrocephalus patients whose cerebrospinal fluid dynamics was investigated using infusion tests involving controllable test-rise of ICP.

Method Cerebral blood flow velocity (FV), ICP and noninvasive arterial blood pressure (ABP) were recorded in 53 patients diagnosed for hydrocephalus. Non-invasive ICP methods were based on: (1) interaction between FV and ABP using black-box model ( $\left.n I C P \_B B\right)$; (2) diastolic FV (nICP_FVd); (3) critical closing pressure ( $\left.n I C P \_C r C P\right)$; (4) transcranial Doppler-derived pulsatility index $\left(n I C P \_P I\right)$. Correlation between rise in ICP $(\triangle \mathrm{ICP})$ and $\triangle \mathrm{nICP}$ and averaged correlations for changes in time between ICP and nICP during infusion test were investigated.

Results From baseline to plateau, all nICP estimators increased significantly. Correlations between $\triangle \mathrm{ICP}$ and $\triangle \mathrm{nICP}$ were better represented by $n I C P \_P I$ and $n I C P \_B B: 0.45$ and
\end{abstract}

D. Cardim

dac69@cam.ac.uk

1 Brain Physics Laboratory, Division of Neurosurgery, Department of Clinical Neurosciences, University of Cambridge, Box 167, Cambridge Biomedical Campus, Cambridge CB2 0QQ, UK

2 Neurosciences Critical Care Unit, Addenbrooke's Hospital, Cambridge University Hospitals NHS Foundation Trust, Cambridge, UK

3 Department of Neurology, University Hospital Aachen, Aachen, Germany

4 Division of Neurosurgery, Addenbrooke's Hospital, Cambridge University Hospitals NHS Foundation Trust, Cambridge, UK

5 Institute of Electronic Systems, Warsaw University of Technology, Warsaw, Poland $0.30(p<0.05) . n I C P \_F V d$ and $n I C P \_C r C P$ presented nonsignificant correlations: $-0.17(p=0.21), 0.21(p=0.13)$. For changes in ICP during individual infusion test $n I C P+P I$, $n I C P \_B B$ and $n I C P P_{-} F V d$ presented similar correlations with ICP: $0.39 \pm 0.40,0.39 \pm 0.43$ and $0.35 \pm 0.41$ respectively. However, $n I C P \_C r C P$ presented a weaker correlation $(R=0.29 \pm 0.24)$.

Conclusions Out of the four methods, nICP_PI was the one with best performance for predicting changes in $\triangle \mathrm{ICP}$ during infusion test, followed by $n I C P_{-} B B$. Unreliable correlations were shown by $n I C P+F V d$ and $n I C P P_{-} C r C P$. Changes of ICP observed during the test were expressed by nICP values with only moderate correlations.

Keywords Non-invasive ICP monitoring · Transcranial Doppler · Cerebral blood flow velocity · CSF infusion test

\section{Introduction}

Intracranial pressure (ICP) monitoring is indicated in a wide range of neurological diseases. However, apart from major trauma and academic neurosurgical centres, it is not commonly part of the clinical management of patients. This scenario is mainly attributed to the invasiveness of the current methods (which require insertion of a catheter into the brain) and their associated risks (infections, brain parenchyma damage and haemorrhages). Such characteristics limit ICP monitoring in several clinical conditions in which ICP would be important: patients with haemorrhagic or ischaemic stroke, mild to moderate traumatic brain injury (TBI), altered mental status or cognitive/psychological disorders, brain tumours and hydrocephalus $[7,8,11]$. As an effort to provide alternatives for ICP monitoring, some methods have been proposed to measure and monitor ICP non-invasively (nICP). 
Transcranial Doppler (TCD), for instance, is primarily a technique for diagnosing various intracranial vascular disorders such as emboli, stenosis or vasospasm [15], but has been broadly utilised for non-invasive ICP monitoring due to its ability to detect changes in cerebral blood flow velocity (FV) derived from ICP variations. TCD has also been used for non-invasive estimation of cerebral perfusion pressure (CPP), based on some parameters derived from FV, such as diastolic flow velocity (FVd) [6] and critical closing pressure (CrCP) [17]. Considering the assumption that CPP equals the difference of arterial blood pressure (ABP) and ICP, nICP can be estimated as $\mathbf{n I C P}=\mathbf{A B P}-\mathbf{n C P P}$.

Infusion test is a diagnostic modality that enables assessment of the cerebrospinal fluid (CSF) dynamics and the compensatory ability of the cranial-spinal fluid system in patients diagnosed with hydrocephalus [18]. The principle of this test is based on a constant infusion rate of artificial CSF into any accessible CSF compartment, which produces a gradual and uncompensated increase in ICP [10]. Our objective in this study was to compare the estimation performances of four TCD-based nICP methods in cohort of hydrocephalus patients whose CSF dynamics were investigated using infusion tests involving controllable test-rise of ICP.

\section{Materials and methods}

\section{Patients}

We retrospectively analysed data collected during the period of 1994-1998 and 2006 at Addenbrooke's Hospital, Cambridge, United Kingdom. The population consisted of 53 patients who undertook an infusion test in order to investigate CSF disturbances. Measurements included ICP, ABP and FV in middle cerebral artery (MCA). The median age of the patients was 55 years [interquartile range (IQR), 66-38 years; 31 men, 22 women].

\section{Infusion test}

An infusion test is a computerised method involving cerebrospinal infusion of artificial CSF (normal saline or Hartman's solution) into the subarachnoid space, based on the traditional constant rate infusion test described by Katzman and Hussey [10]. For this purpose, one spinal needle (18 G, 3.50 in.) is inserted between the lumbar L3/L4 or L4/L5 vertebrae, and it is used for both pressure measurements and fluid infusion. This needle is connected to a pressure transducer via a stiff saline-filled tube and to an infusion device (Alaris ${ }^{\circledR}$ GH Plus Syringe Pump; Carefusion, Basingstoke, UK) with a $50-\mathrm{mL}$ syringe (BD, Oxford, UK), mounted on a trolley containing a pressure monitor (Simonsen and Will, Sidcup, England) connected to a computer [5]. The threshold of the opening pressure is $13 \mathrm{mmHg}$ and the infusion rate is $1 \mathrm{~mL} / \mathrm{min}$ when the pressure is higher than the threshold. Conversely, the infusion rate is $1.5 \mathrm{~mL} / \mathrm{min}$ when the pressure is lower than the threshold. The start of infusion is initiated after baseline recording (10 $\mathrm{min})$, whereas the end of the infusion is signalled by the achievement of a steady-state ICP plateau. If ICP rises above $40 \mathrm{mmHg}$, the infusion needs to be discontinued to avoid excessive elevation of the pressure above the safe clinical limits [18]. After cessation of the infusion, ICP is continuously recorded until it decreases to a steady baseline level [5].

\section{Data acquisition}

The ICP measurements were made with the patient placed in a lateral position (left or right), considering the foramen of Monro as the zero calibration reference. TCD ultrasonography (Neuroguard; Medasonics, Fremont, CA, USA) was used for monitoring of blood FV in the MCA, through a 2$\mathrm{MHz}$ probe fixed on the cranium by using a commercially available headband. ABP was recorded non-invasively by using a Finapres ${ }^{\circledR}$ finger cuff (Ohmeda, Englewood, CO, USA) positioned at the level of the heart. Analogue outputs of ICP and ABP from the pressure monitors and the TCD monitor were connected to an analogue-to-digital converter (DT2814; Data Translation, Marlboro, CA, USA) fitted into an IBM-compatible laptop computer (Amstrad ALT 386 SX; Amstrad, Brentwood, UK). Data were sampled $(50 \mathrm{~Hz})$, digitised and stored on the hard disk using software for waveform recording (WREC; W. Zabolotny, Warsaw University of Technology, Warsaw, Poland). The recorded signals were then analysed using $\mathrm{ICM}+$ software (Cambridge Enterprise, http://www.neurosurg.cam.ac.uk/ icmplus). Informed consent was obtained from all individual participants included in the study (or their next of kin) for the use of collected data for research purposes. The infusion test is a routine clinical investigation in the Hydrocephalus Clinic, Addenbrooke's Hospital, Cambridge, with no ethical approval required. The data were analysed anonymously as a part of a clinical audit.

\section{Non-invasive ICP methods}

The four methods used for nICP estimation in this study were:

1. Schmidt et al. [14] "black-box" (BB) model (nICP BB): nICP is obtained from a mathematical "black-box" model based on the presumed transformation between ABP and ICP waveforms. Coefficients of these transformations are derived from the database of real ABP and ICP recordings. Similar linear transformation is built, using the same database between FV and ABP. Then, the model assumes a linear relationship between $\mathrm{ABP}$ and $\mathrm{FV}$ and $\mathrm{ABP}$ to 
ICP transformations. Multiple regression coefficients are calculated. Finally, for each prospective study, ICP is calculated using ABP to ICP transformation, formed from ABP to FV transformation transposed using precalculated regression coefficients. Non-invasive ICP estimation using this method was performed using a plugin developed for ICM+ software.

2. Czosnyka et al. [6] ( $\left.n I C P \_F V d\right)$ : for this method, based on the diastolic flow velocity for the estimation of $\mathrm{nCPP}, \mathrm{nICP}$ was calculated as the difference between $\mathrm{ABP}$ and $\mathrm{nCPP}$ $(\mathrm{nICP}=\mathrm{ABP}-\mathrm{nCPP})$. The equation for $\mathrm{nCPP}$ estimation is:

$n C P P=A B P \times \frac{F V d}{F V m}+14$

FVm and FVd represent mean and diastolic flow velocity, respectively.

3. Varsos et al. [17] ( $\mathrm{ICP} C \mathrm{CrCP})$ : this method also calculates nICP based on nCPP, in this case specifically using the concept of critical closing pressure $(\mathrm{CrCP})$. The equation for nCPP estimation is:

$n C P P=A B P \times\left[0.734-\frac{0.266}{\sqrt{(C V R \times C a \times H R \times 2 \pi)^{2}+1}}\right]-7.026$

CVR represents cerebral vascular resistance, $C a$ denotes arterial compliance of the cerebral bed and $H R$ expresses heart rate (beats/s), with $\mathrm{ABP}$ and $\mathrm{FV}$ as the required measurements. Constant coefficients $(0.734$, $0.266,7.026)$ are derived from analysis of database of 232 TBI retrospective cases [17].

4. nICP_PI: nICP estimation based on TCD-derived pulsatility index was based on the linear regression among known values of ICP and pulsatility index (PI) from a population cohort of 292 TBI patients. The regression equation was based on data analysed by Budohoski et al. [2] and given by:

$n I C P=4.47 \times P I+12.68$

$P I=\frac{F V_{s}-F V_{d}}{F V_{m}}$

$F V s, F V d$ and $F V m$ represent systolic, diastolic and mean flow velocity.

\section{Statistical analysis}

Statistical analysis of the data was conducted with OriginPro statistical software (version 8; OriginLab, Northampton, MA, USA). Data were tested for normal distribution using ShapiroWilk test. The analysis included Spearman correlations between mean $\triangle \mathrm{ICP}$ and $\triangle \mathrm{nICP}$ and averaged correlations for variations of nICP across time during ICP increase. The $\Delta$ value (magnitude) was considered the difference between plateau and baseline mean values in each recording during infusion. $R$ represents the Spearman correlation coefficient, with the level of significance set at 0.05 . The Bland-Altman method was used to determine the agreement between measured ICP and the different nICP methods, with their respective $95 \%$ CI for prediction of ICP and bias for baseline and plateau periods. The confidence interval represents the range of values around the bias (difference between mean values of nICP and ICP), in which data can be found with a significance level of 0.05. In addition, Mann-Whitney test was used to assess whether nICP samples originate from the same distribution as ICP for both baseline and plateau phases of infusion test. Wilcoxon test was applied to assess whether there was any significant difference between baseline and plateau phases for each estimator and calculated variables.

\section{Results}

Table 1 presents all estimated physiological variables used for nICP estimations, at baseline and plateau phases, and their $\Delta$ correlations with $\triangle \mathrm{ICP}$. Table 2 presents comparisons among non-invasive methods adopted in this study.

Median values of ICP and nICP are presented with their respective interquartile ranges (IQRs) in mmHg. Mann-Whitney test revealed significantly different distributions between all nICP estimators and ICP for baseline; for plateau phase only $n I C P \_B B$ and $n I C P \_P I$ presented such differences. Wilcoxon test revealed significantly different distributions between baseline and plateau nICPs and ICP.

Regarding confidence intervals (Bias $\pm 95 \% \mathrm{CI}$ ), $n I C P \_B B$ showed $15.33 \pm 4.46 \mathrm{mmHg} ; n I C P \_F V d$ showed 25.19 $\pm 11.90 \mathrm{mmHg}$; $n I C P \_C r C P$ showed $15.09 \pm 11.12 \mathrm{mmHg}$ and $n I C P$ PI showed $10.58 \pm 8.91 \mathrm{mmHg}$. During plateau every method presented increased CI: $19.21 \pm-7.35 \mathrm{mmHg}$ for $n I C P \_B B ; 29.97 \pm 1.66 \mathrm{mmHg}$ for $n I C P \_F V d ; 17.80 \pm$ $-2.53 \mathrm{mmHg}$ for $n I C P C r C P$ and $19.07 \pm-6.18 \mathrm{mmHg}$ for nICP PI.

Correlations between $\triangle \mathrm{ICP}$ and $\triangle \mathrm{nICP}$ were better represented by $n I C P$ PI and $n I C P B B$, with $0.45(p=0.0007)$ and $R=0.30$ ( $p=0.03)$, respectively. The other methods presented inferior and non-significant correlations: $-0.17(p=0.21)$, $0.21(p=0.13)$ for $I C P n_{-} F V d, n I C P \_C r C P$, respectively. 
Table 1 Median values (IQR), $95 \%$ CI for prediction of ICP and bias (in $\mathrm{mmHg}$ ) are described for baseline and plateau phases. Spearman correlation between $\triangle \mathrm{ICP}$ vs $\triangle \mathrm{nICP}$ and averaged correlation across time during ICP increase $(n=53)$. At the 0.05 level, baseline and plateau distributions of nICPs and ICP are significantly different

\begin{tabular}{|c|c|c|c|c|c|c|c|c|}
\hline \multirow[t]{2}{*}{ Method } & \multicolumn{3}{|l|}{ Baseline } & \multicolumn{3}{|l|}{ Plateau } & \multirow{2}{*}{$\begin{array}{l}R \\
(\Delta \mathrm{ICP} \text { vs } \triangle \mathrm{nICP})\end{array}$} & \multirow{2}{*}{$\begin{array}{l}R \\
\text { (time domain) }\end{array}$} \\
\hline & Median (IQR) & $95 \% \mathrm{CI}$ & Bias & Median (IQR) & $95 \% \mathrm{CI}$ & Bias & & \\
\hline$n I C P \_B B$ & $10.76(15.08-7.30)^{\mathrm{a}}$ & 15.33 & $4.46^{\mathrm{b}}$ & $14.86(20.1-11.26)^{\mathrm{a}}$ & 19.21 & $-7.35^{\mathrm{b}}$ & $0.30^{\mathrm{c}}$ & $0.39 \pm 0.43$ \\
\hline$n I C P \_F V d$ & $16.97(22.56-11.64)^{\mathrm{a}}$ & 25.19 & $11.90^{\mathrm{b}}$ & $21.74(32.85-14.15)$ & 29.97 & 1.66 & -0.17 & $0.35 \pm 0.41$ \\
\hline$n I C P \_C r C P$ & $18.34(20.38-14.89)^{\mathrm{a}}$ & 15.09 & $11.12^{\mathrm{b}}$ & $19.65(23.80-16.92)$ & 17.80 & $-2.53^{\mathrm{b}}$ & 0.21 & $0.29 \pm 0.24$ \\
\hline$n I C P \_P I$ & $16.57(17.46-16.06)^{\mathrm{a}}$ & 10.58 & $8.91^{\mathrm{b}}$ & $17.12(17.73-16.40)^{\mathrm{a}}$ & 19.07 & $-6.18^{\mathrm{b}}$ & $0.45^{\mathrm{c}}$ & $0.39 \pm 0.40$ \\
\hline$I C P$ & $7.74(11.06-2.95)$ & - & - & $22.13(29.77-16.41)$ & - & - & - & - \\
\hline
\end{tabular}

a At the 0.05 level, distributions between nICP and ICP are significantly different

$\mathrm{b}$ The population mean is significantly different with the test mean (zero)

c Spearman correlation coefficient is significant at the 0.05 level

Regarding variations of nICP in time domain during ICP increase $n I C P \quad P I, n I C P B B$ and $n I C P$ FVd presented similar averaged correlations, $0.39 \pm 0.40,0.39 \pm 0.43$ and $0.35 \pm 0.41$, respectively. However, $n I C P C r C P$ presented a smaller correlation $(R=0.29 \pm 0.24)$. To demonstrate such variations and correlation between nICP and ICP in time, examples of a good and a poor recording of nICP with the four investigated methods when ICP changed considerably during the infusion test are showed in Figs. 1 and 2, respectively.

\section{Discussion}

TCD has been used to estimate nICP in several conditions; however, their relative accuracy varies between different types of intracranial hypertension: vasogenic, CSF circulatory or secondary to brain volumetric changes (oedema, contusion, haematoma, etc.). Schmidt et al. [14] reported a CI for prediction of $12.8 \mathrm{mmHg}$, and Cardim et al. [3] a CI of $9.94 \mathrm{mmHg}$ for $n I C P B B$. Kashif et al. [9], also using a model based on $\mathrm{ABP}$ and $\mathrm{FV}$, reported $15 \mathrm{mmHg}$ (standard deviation of error (SDE) of $7.6 \mathrm{mmHg}$ ). Using TCD-derived PI, Bellner et al. [1] and Cardim et al. [3] reported a $95 \% \mathrm{CI}$ for prediction of $4.2 \mathrm{mmHg}$ and $9.62 \mathrm{mmHg}$, respectively. Non-invasive ICP methods based on the concepts of $\mathrm{FVd}$ and $\mathrm{CrCP}$ were also assessed and presented with CI of $14.62 \mathrm{mmHg}$ and $9.19 \mathrm{mmHg}$, respectively [3]. For the moment, these confidence intervals have mainly been described for traumatic brain injury patients, with the exception of PI, which reflected a cohort of patients with several intracranial disorders [1].

The assessment of TCD-based nICP estimators during infusion tests revealed a limited accuracy of assessment ICP non-invasively and discrepancy in estimation of ICP. Of the four studied estimators, relative changes in mean ICP were better associated with estimated ICP using only two methods. Best scenario was obtained with the method based on TCD pulsatility index, followed by the mathematical black-box model, whereas the other methods did not present satisfactory correlation with measured ICP.
Table 2 Median (IQR) values for all physiological variables estimated during baseline and plateau phases, with their respective $\Delta$ correlations with $\triangle \mathrm{ICP}(R)$

\begin{tabular}{llllll}
\hline Variable & Baseline & Plateau & $p$ value & $R(\Delta \mathrm{ICP})$ & $R(\Delta \mathrm{ABP})$ \\
\hline $\mathrm{ICP}$ & $7.77(11.06-2.95)$ & $22.13(29.77-16.41)^{\mathrm{a}}$ & $2.2 \times 10^{-16}$ & - & 0.1 \\
$\mathrm{ABP}$ & $89.68(101.5-78.23)$ & $96.08(121-87.07)^{\mathrm{a}}$ & $5.6 \times 10^{-6}$ & 0.11 & - \\
$\mathrm{HR}$ & $67.86(77.96-61.98)$ & $70.12(79-60.83)$ & 0.39 & 0.15 & $0.28^{\mathrm{b}}$ \\
$\mathrm{CPP}$ & $82(98.67-73.27)$ & $79.40(96.53-67.14)^{\mathrm{a}}$ & $7.9 \times 10^{-4}$ & $-0.38^{\mathrm{b}}$ & $0.82^{\mathrm{b}}$ \\
$\mathrm{FV}$ & $54.2(66.27-42.47)$ & $49.76(61.92-39.07)^{\mathrm{a}}$ & $8.4 \times 10^{-6}$ & 0.14 & -0.20 \\
FV $_{\mathrm{s}}$ & $81.61(101.6-66.76)$ & $79.97(98.65-63.35)$ & 0.07 & -0.03 & -0.21 \\
$\mathrm{FV}_{\mathrm{d}}$ & $33.17(43.86-27.32)$ & $31.93(40.61-23.57)^{\mathrm{a}}$ & $1.4 \times 10^{-6}$ & -0.22 & 0.21 \\
PI & $0.84(1.05-0.76)$ & $0.99(1.13-0.83)^{\mathrm{a}}$ & $1.1 \times 10^{-9}$ & $0.45^{\mathrm{b}}$ & -0.04 \\
$\mathrm{CVR}$ & $1.64(1.95-1.22)$ & $1.55(2.05-1.13)$ & 0.56 & -0.21 & $0.62^{\mathrm{b}}$ \\
\hline
\end{tabular}

${ }^{\text {a }}$ At the 0.05 level, distributions between baseline and plateau are significantly different

b Spearman correlation coefficient is significant at the 0.05 level 

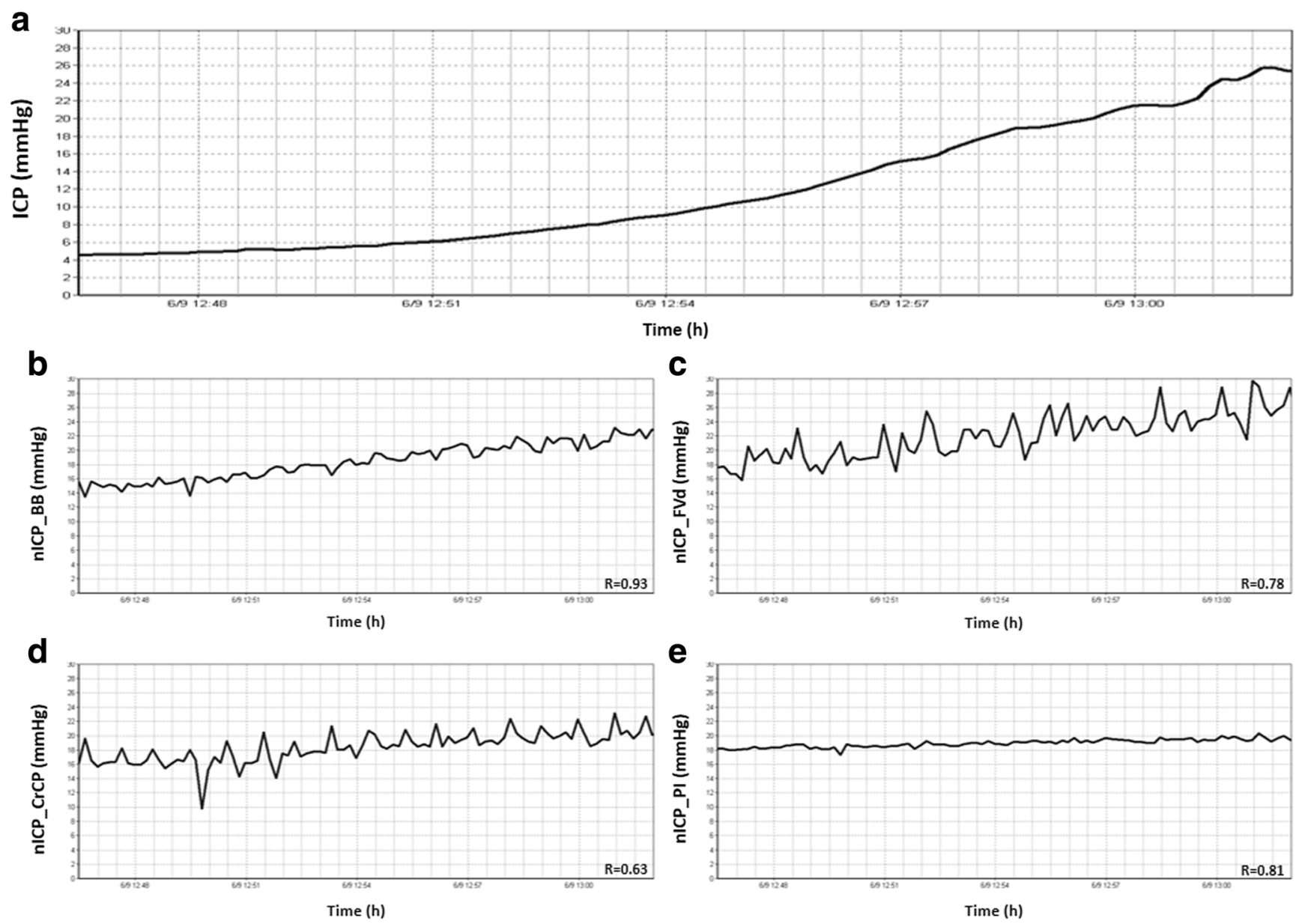

Fig. 1 Example of good recording of nICP with four investigated methods when ICP changed considerably during the infusion test. a ICP; b $n I C P \_B B ; \mathbf{c} n I C P \_F V d ; \mathbf{d} n I C P \_C r C P ; \mathbf{e} n I C P \_P I . R$ represents

Considering trends of measured ICP in time, all nICP methods presented better performance and overall correlations were more balanced among them.

Considering absolute values of ICP, analysis showed significantly different distributions between all nICP methods and ICP for baseline, whereas during the plateau phase $n I C P \_F d$ and $n I C P \_C r C P$ had the same distributions as ICP. This indicates that, with exception of these cases, the nICP estimators were not able to accurately predict the absolute measure of ICP during these phases of the infusion test.

Nevertheless, all paired comparisons between estimators for baseline and plateau showed significant differences, demonstrating that infusion test did produce a significant increase in nICP for every estimator. Although significant within the same method, these differences were disproportional when comparing measured ICP and nICP estimations. Such disproportion was reflected in the confidence intervals for prediction found for baseline and plateau phases. In regards to bias, a non-significant difference between non-invasive and invasive methods is desirable,

correlation coefficient between ICP and $\mathrm{nICP}\left(R=0.93\right.$ for $n I C P \_B B$; $R=0.78$ for $n I C P \quad F V d ; R=0.63$ for $n I C P C r C P ; R=0.81$ for $n I C P-P I)$

which means that both methods are not different in rendering mean ICP values. In this case, with exception of $n I C P_{-} F V d$ during plateau phase, all estimators presented significant biases.

Regarding correlations between $\triangle \mathrm{ICP}$ and $\triangle \mathrm{nICP}$, such results demonstrate the ability of the nICP methods to detect the magnitude of changes in measured ICP. Even though considered moderate correlations, nICP_PI and $n I C P \_B B$ were the only estimators to present significant levels. For detection of trends in time, which does not consider ICP as a numeric value but the behaviour of the methods during an ICP increase, the methods also displayed moderate averaged correlations. They showed better agreement for $n I C P P_{-} P I, n I C P \_B B$ and $n I C P \_F V d$, and a weaker correlation for $n I C P P_{C} C r C P$. Figures 1 and 2, respectively, represent good and poor examples of correlations in time among ICP and nICP estimators.

As observed, nICP estimation differed mostly in terms of prediction of absolute values of ICP during infusion tests and to a lesser extent in terms of detection of dynamic changes. 

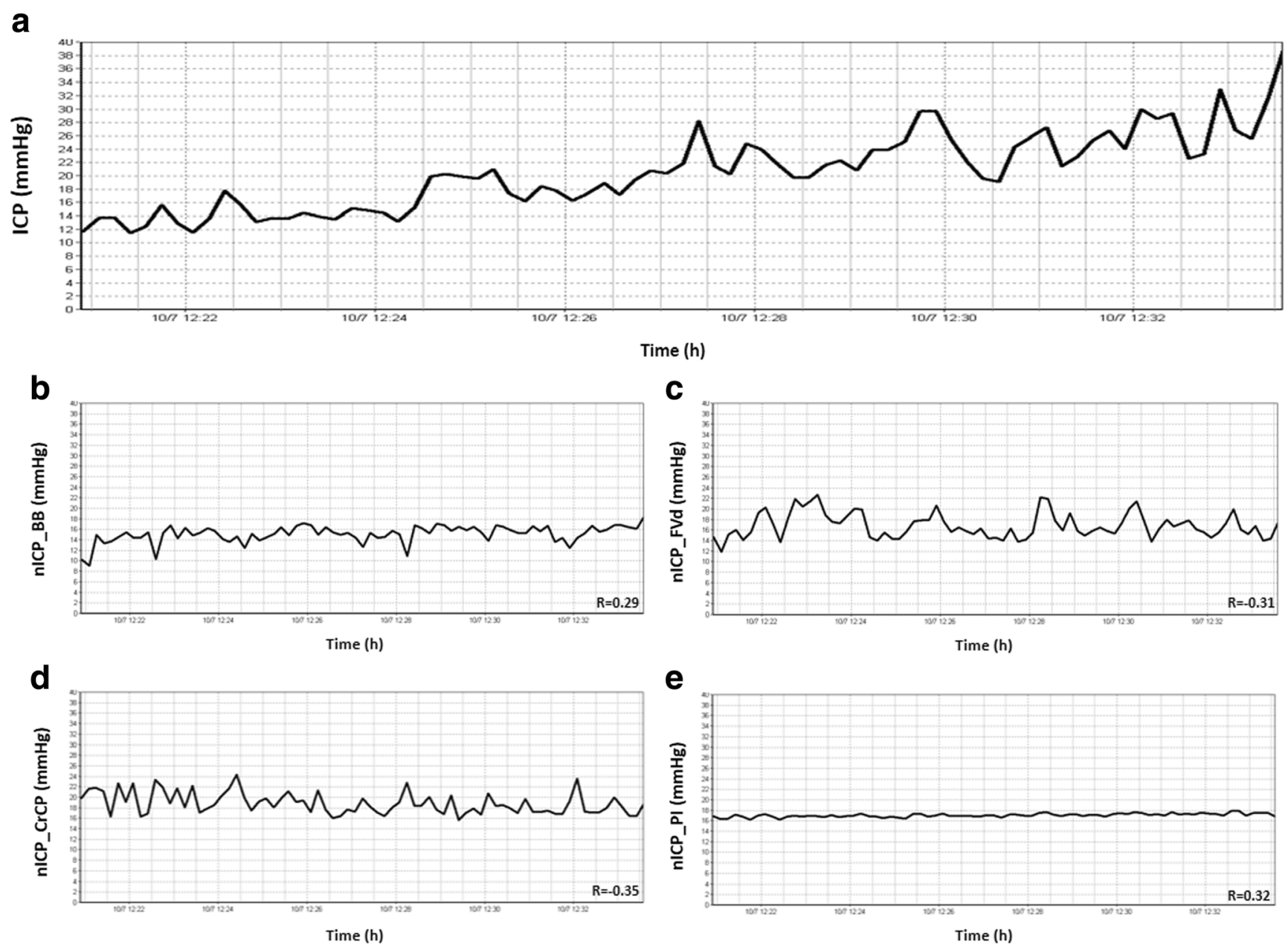

Fig. 2 Example of poor recording of nICP with four investigated methods when ICP changed considerably during the infusion test. a ICP; b $n I C P \_B B ; \mathbf{c} n I C P \_F V d ; \mathbf{d} n I C P \_C r C P ; \mathbf{e} n I C P \_P I . R$ represents

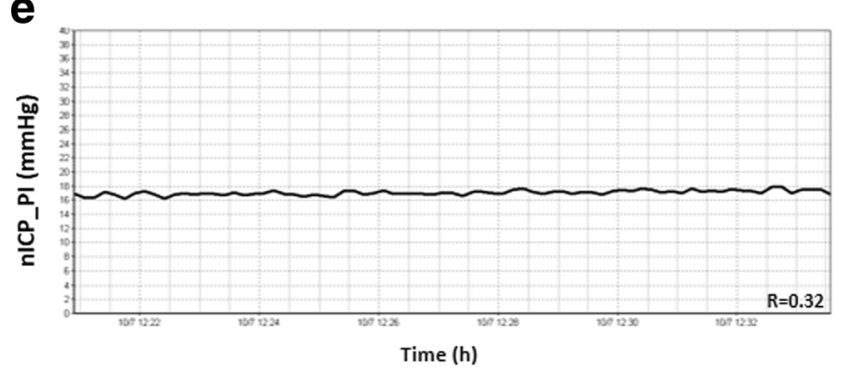

correlation coefficient between ICP and nICP $\left(R=0.29\right.$ for $n I C P \_B B$; $R=-0.31$ for $n I C P F V d ; R=-0.35$ for $n I C P C r C P ; R=0.3 \overline{2}$ for nICP_PI)

Different nICP accuracies may be explained by each method's specific characteristics. The $n I C P \_B B$ method reflects ABP waveform being constantly modified by TCD characteristics, and then is mostly susceptible to changes of vasogenic origin (such as CVR) and consequently cerebral blood flow. The $n I C P \_F V d$ method (Eq. 1) is mostly modulated by the factor $\mathrm{FVd} / \mathrm{FVm}$ and ABP. The $n I C P \_C r C P$ method (Eq. 2) is modulated by changes in CVR and also by ABP. For $n I C P \_P I$, any changes in the components of flow velocity (FVm, FVs and $\mathrm{FVd}$ ) are reflected in the PI calculation and consequently in the nICP estimation.

The nature of ICP elevation during infusion test is attributed to an increase in CSF circulation due to a direct addition of volume into the CSF space. TCD ultrasonography, however, is a technique mainly capable of detecting cerebrovascular changes in the arterial bed [15] (in our case, specifically the MCA). Thus, theoretically, it would be expected that changes in ICP estimated with TCD-based methods would present better

accuracy if they were of vasogenic origin, rather than caused by variations of pressure in the CSF compartment.

This can be exemplified by the fact that in specific cases where changes of ICP related to vasogenic fluctuations (plateau waves, B waves) overlapped the rise related to CSF infusion, the time-trend correlation between real and estimated ICP seemed to be remarkably better (as seen in Fig. 3), even with reliable replications of vasogenic waves patterns.

The way fluctuations in CSF circulation are transmitted to cerebral arterial bed might help understand the low accuracies presented by TCD methods during infusion test. In these circumstances, the rise in ICP originated from increased CSF circulation has direct and indirect influences on cerebral haemodynamics. The direct influence is represented by changes in CPP, altered directly by ICP. On the other hand, ICP has an indirect effect on cerebral haemodynamics, via changes in systemic haemodynamics. The changes in ABP observed during 

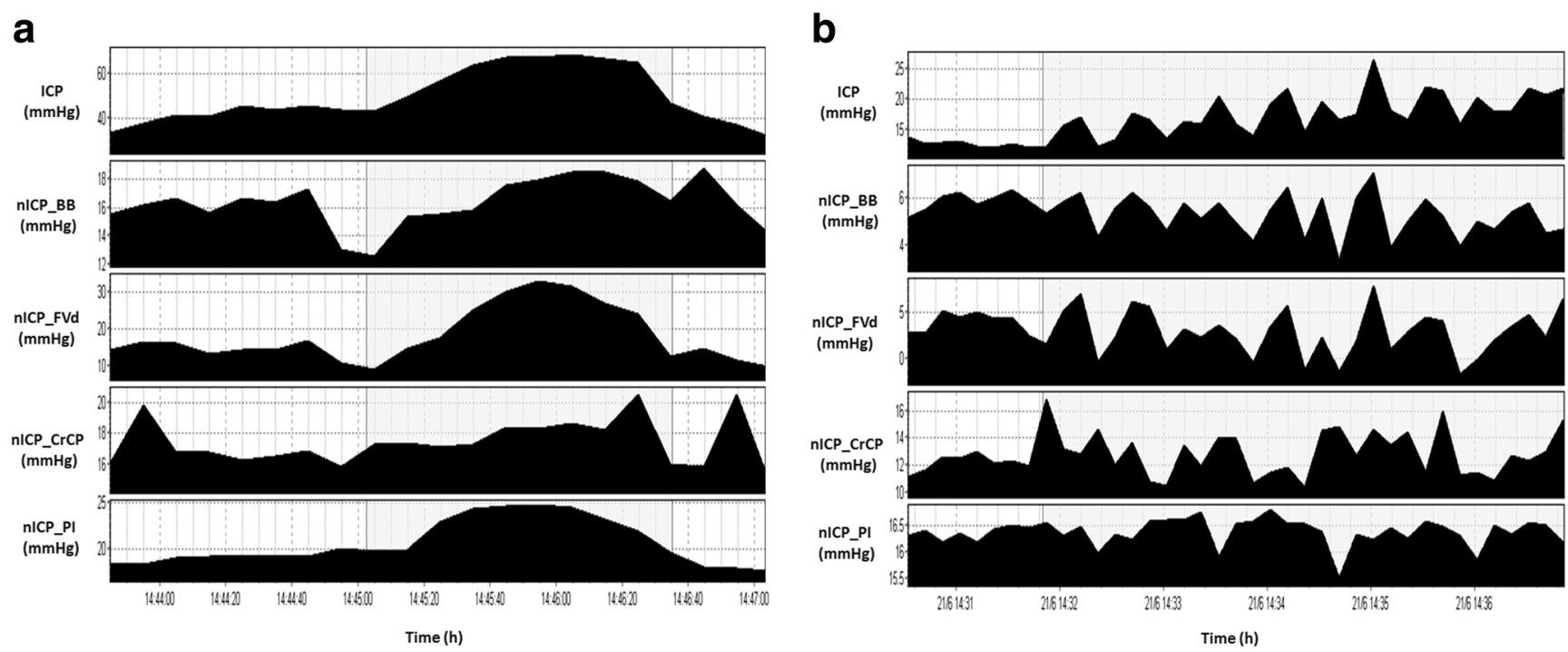

Fig. 3 Example of vasogenic waves during infusion test. a Shadowed area represents a plateau wave of ICP. b Shadowed area represents B waves of ICP. It is possible to observe that at least for trends in time, there were good concordance between ICP and nICP methods

infusion tests are associated with an early Cushing response [13], in which rising ICP yields an increase in ABP. Therefore, transmission of fluctuations in CSF circulation to the cerebral arterial bed are primarily given by a decrease in CPP and secondarily by an increase in ABP.

In regard to haemodynamic and TCD-derived cerebrovascular variables used for the method's estimations (ABP, HR, FV, FVs, FVd, PI and CVR), HR, FVs and CVR did not differ between plateau and baseline phases. Within the variables which presented significant difference, only changes in CPP and PI ( $\triangle \mathrm{CPP}$ and $\Delta \mathrm{PI})$ were significantly correlated with changes in ICP. This finding is in agreement with results obtained by Bellner et al. [1], in which PI was strongly correlated with changes in ICP. In our case, however, PI presented only a moderate correlation with ICP, and was mainly modulated by FVd, as FVs did not present significant changes between baseline and plateau.

In this study, changes in ABP were significantly correlated to changes in CVR. This signifies that although such changes were not significant between baseline and plateau phases, this secondary increase in ABP produced by an early Cushing response caused minor vasogenic changes in cerebral haemodynamics [13]. This could have contributed to the better accuracy in cases which patients presented changes in ICP of vasogenic origin (Fig. 3).

Consequently, the low accuracy for prediction of ICP observed for TCD-based methods might be related to the nature of ICP elevation during infusion tests. Although increased CSF circulation is able to produce secondary changes on cerebral haemodynamics, this might not be the ideal manner to produce global changes of vasogenic origin that could be detected more accurately by TCD. Therefore, under these conditions, TCD cannot be considered a suitable technique for nICP estimation in conditions of increased CSF circulation.

In conclusion, changes of ICP observed during the test were expressed by nICP values with only moderate correlations. Vasogenic components of ICP seemed to be easier to estimate with TCD, than component related to increased CSF circulation. In this context, out of the four methods assessed, $n I C P \_P I$ was the one with best performance for predicting changes in $\triangle \mathrm{ICP}$ during infusion test, followed by $n I C P \_B B$. Methods based on FVd and $\mathrm{CrCP}$ showed unreliable correlations.

\section{Limitations}

An intrinsic limitation of this study is that it considers analysis of retrospective data, collected at two different time points (1994-1998 and 2006). The majority of data was collected in the first period ( $n=39,73.6 \%)$; nevertheless, the methodology and equipment used did not differ between the collection points. However analysis of retrospective data may evidence downsides of the study design, such material is unique and contains important information about the cerebrovascular dynamics of normal pressure hydrocephalus (NPH) patients during CSF infusion tests, which may be useful in different scenarios and applications.

A well-known limitation in studies approaching cerebral blood flow velocity measured with TCD is the inter- and intra-operator variability, as demonstrated by McMahon et al. [12]. Here, we attempted to minimise it by allowing only two experienced operators (M.C. and Z.C.) to collect the data. 
It is then assumed for this study that such variability was minimal.

A potential limiting factor to this study could be the non-invasive measurement of ABP. Although the response bandwidth obtained with Finapres ${ }^{\circledR}$ is adequate to register the ABP waveform with reliable accuracy, the supporting applications in which waveformdependent indices are extracted [4] (such as in $n I C P \_B B$ method), relative accuracy in terms of mean values of ABP did not show to be very precise. In a previous study, Stokes et al. [16] performed a comparison of invasive and non-invasive ABP measurements (specifically using Ohmeda Finapres ${ }^{\circledR}$ 2300), and found an inter-individual variability in absolute pressure readings. In our case, this could promote a misestimation for $n I C P \_F V d$ and $n I C P P_{-} C r C P$, which present ABP as multiplier in the formula.

Another limiting factor could be the origin of the methods used for nICP estimations, as all of them were derived from cohorts of traumatic brain injury patients. Different physiological mechanisms leading to ICP fluctuations in TBI and hydrocephalus might play a significant role in the way ICP was estimated.

\section{Compliance with ethical standards}

Funding Cambridge Commonwealth, European and International Trust Scholarship, University of Cambridge provided financial support in the form of Scholarship funding for D.C. Woolf Fisher Trust provided financial support in the form of Scholarship funding for J.D. Gates Cambridge Trust provided financial support in the form of Scholarship funding for X.L. CNPQ provided financial support in the form of Scholarship funding for B.C.T.C. (Research Project 203792/2014-9). Alexander-von-Humboldt Foundation provided financial support in the form of a FeodorLynen scholarship funding for C.H. NIHR Brain Injury Healthcare Technology Co-operative, Cambridge, UK provided financial support in the form of equipment funding for D.C. and M.C. The sponsors had no role in the design or conduct of this research.

Conflict of interest ICM + software is licensed by the University of Cambridge, Cambridge Enterprise Ltd. MC and PS have a financial interest in a part of its licensing fee. The remaining authors certify that they have no affiliations with or involvement in any organization or entity with any financial interest (such as honoraria; educational grants; participation in speakers' bureaus; membership, employment, consultancies, stock ownership, or other equity interest; and expert testimony or patent-licensing arrangements). All authors certify that they have no non-financial interest (such as personal or professional relationships, affiliations, knowledge or beliefs) in the subject matter or materials discussed in this manuscript.

Ethical approval The infusion test is a routine clinical investigation in the Hydrocephalus Clinic, Addenbrooke's Hospital, Cambridge, with no ethical approval required. The data were analysed anonymously as a part of a clinical audit.
Informed consent Informed consent was obtained from all individual participants included in the study (or their next of kin) for the use of collected data for research purposes.

Open Access This article is distributed under the terms of the Creative Commons Attribution 4.0 International License (http:// creativecommons.org/licenses/by/4.0/), which permits unrestricted use, distribution, and reproduction in any medium, provided you give appropriate credit to the original author(s) and the source, provide a link to the Creative Commons license, and indicate if changes were made.

\section{References}

1. Bellner J, Romner B, Reinstrup P, Kristiansson KA, Ryding E, Brandt L (2004) Transcranial Doppler sonography pulsatility index (PI) reflects intracranial pressure (ICP). Surg Neurol 62:45-51

2. Budohoski KP, Schmidt B, Smielewski P, Kasprowicz M, Plontke R, Pickard JD, Klingelhöfer J, Czosnyka M (2012) Non-invasively estimated ICP pulse amplitude strongly correlates with outcome after TBI. Acta Neurochir Suppl 114:121-125

3. Cardim D, Robba C, Donnelly J, Bohdanowicz M, Schmidt B, Damian M, Varsos GV, Liu X, Cabeleira M, Frigieri G, Cabella B, Smielewsky P, Mascarenhas S, Czosnyka M (2015) Prospective study on non-invasive assessment of ICP in head injured patients: comparison of four methods. J Neurotrauma. doi:10. 1089/neu.2015.4134

4. Chin KY, Panerai RB (2012) Comparative study of Finapres devices. Blood Press Monit 17(4):171-178

5. Czosnyka ZH, Czosnyka M, Whitfield PC, Donovan T, Pickard JD, Milhorat TH, Selman WR, Gjerris F, Juhler M (2002) Cerebral autoregulation among patients with symptoms of hydrocephalus. Neurosurgery 50(3):526-533

6. Czosnyka M, Matta BF, Smielewski P, Kirkpatrick PJ, Pickard JD (1998) Cerebral perfusion pressure in head-injured patients: a noninvasive assessment using transcranial Doppler ultrasonography. J Neurosurg 88:802-808

7. DeWitt DS, Prough DS (2003) Traumatic cerebral vascular injury: the effects of concussive brain injury on the cerebral vasculature. J Neurotrauma 20(9):795-825

8. Hoge CW, McHurk D, Thomas JL, Cox AL, Engel CC, Castro CA (2008) Mild traumatic brain injury in U.S. soldiers returning from Iraq. N Engl J Med 358(5):453-463

9. Kashif FM, Verghese GC, Novak V, Czosnyka M, Heldt T (2012) Model-based noninvasive estimation of intracranial pressure from cerebral blood flow velocity and arterial pressure. Sci Transl Med 4(129):129ra44

10. Katzman R, Hussey F (1970) A simple constant-infusion manometric test for measurement of CSF absorption. I. Rationale and method. Neurology 20(6):534-544

11. Marmarou A (2007) A review of progress in understanding the pathophysiology and treatment of brain edema. Neurosurg Focus 22(5):E1

12. McMahon CJ, McDermott P, Horsfall D, Selvarajah JR, King AT, Vail A (2007) The reproducibility of transcranial Doppler middle cerebral artery velocity measurements: implications for clinical practice. Br J Neurosurg 21(1):21-27

13. Schmidt EA, Czosnyka Z, Momjian S, Czosnyka M, Bech RA, Pickardl JD (2005) Intracranial baroreflex yielding an early Cushing response in human. Acta Neurochir Suppl 95:253-256

14. Schmidt B, Klingelhofer J, Md J, Schwarze JJ, Sander D, Wittich I (1997) Noninvasive prediction of intracranial pressure curves using 
transcranial Doppler ultrasonography and blood pressure curves. Stroke 28:2465-2472

15. Sloan MA, Alexandrov AV, Tegeler CH, Spencer MP, Caplan LP, Feldmann E, Wechsler LR, Newell DW, Gomez CR, Babikian VL, Lefkowitz D, Goldman RS, Armon C, Hsu CY, Goodin DS (2004) Assessment: transcranial Doppler ultrasonography: report of the Therapeutics and Technology Assessment Subcommittee of the American Academy of Neurology. Neurology 62(9):1468-1481

16. Stokes DN, Clutton-Brock T, Patil C, Thompson JM, Hutton P (1991) Comparison of invasive and non-invasive measurement of continuous arterial pressure using the Finapres. Br J Anaesth 67(1):26-35

17. Varsos GV, Kolias AG, Smielewski P, Brady KM, Varsos VG, Hutchinson PJ, Pickard JD, Czosnyka M (2015) A noninvasive estimation of cerebral perfusion pressure using critical closing pressure. J Neurosurg 123(3):638-648

18. Weerakkody RA, Czosnyka M, Schuhmann MU, Schmidt E, Keong N, Santarius T, Pickard JD, Czosnyka Z (2011) Clinical assessment of cerebrospinal fluid dynamics in hydrocephalus. Guide to interpretation based on observational study. Acta Neurol Scand 124(2):85-98

\section{Comment}

Cardim and colleagues retrospectively analysed four different noninvasive methods to assess ICP during CSF infusion test in 53 patients. Even though this is a retrospective study with the well-known limitations of this study design and two of the four methods assessed demonstrated unreliable correlations to the real ICP, the study is worth being published in my opinion, since it would be of high importance if a way of noninvasively assessing ICP could be found. Hopefully, this paper encourages even more study groups to deal with this challenge.

Marcus Reinges

Giessen, Germany 